Décadrages Décadrages

cinéma, à travers champs Cinéma, à travers champs

10 | 2007

La trilogie de Dieu de João César Monteiro

\title{
Mon identité polonaise : Comme des voleurs (à l'Est) de Lionel Baier
}

Laura Legast

\section{(2) OpenEdition}

1 Journals

Édition électronique

URL : https://journals.openedition.org/decadrages/428

DOI : $10.4000 /$ decadrages. 428

ISSN : 2297-5977

Éditeur

Association Décadrages

Édition imprimée

Date de publication : 10 avril 2007

Pagination : 110-114

ISBN : 978-2-9700582-5-0

ISSN : 2235-7823

Référence électronique

Laura Legast, « Mon identité polonaise : Comme des voleurs (à l'Est) de Lionel Baier », Décadrages [En ligne], 10 | 2007, mis en ligne le 10 décembre 2012, consulté le 03 avril 2022. URL : http://

journals.openedition.org/decadrages/428; DOI : https://doi.org/10.4000/decadrages.428

(B) Décadrages 

lisant Garçon stupide (2004) sans renoncer pour autant à l'ancrage du récit dans sa propre biographie. Son dernier long métrage, Comme des voleurs (à l'Est) (2006), propose une démarche très personnelle, s'appuyant sur les particularités des films précédents tout en les radicalisant. En quatre films, ce jeune réalisateur pose les bases d'un cinéma à la première personne, s'inscrivant ainsi dans le genre du journal filmé.

\section{Entre la biographie et la fiction: l'autofiction}

On l'aura compris, le mode autobiographique est le point de départ et le dénominateur commun de l'œuvre de Baier. Dans ses deux premiers documentaires, la pratique du cinéaste s'apparente à celle du journal filmé. Avec un matériel de prise de vue et de son extrêmement léger, avec une équipe de tournage minimale, Baier, s'impliquant personnellement dans ces longs métrages, va à la rencontre de ses sujets caméra au poing: son père pour l'un, la Gay Pride de Sion, ses organisateurs et participants, pour l'autre. Deux films à travers lesquels il part à la recherche de son identité et de ses choix de vie. Avec Celui au pasteur, il cherche à s'affranchir de la figure d'un père tout-puissant, patriarche familial et pasteur de métier. Avec La parade, il affirme publiquement son homosexualité et tente de clarifier sa position au sein de la communauté gay.

Même si Garçon stupide repose sur un contrat de lecture d'ordre fictionnel, il est difficile de ne pas faire le lien avec les films précédents et la biographie de l'auteur. Il y a d'abord le personnage de Loïc, jeune homosexuel lausannois paumé, à la recherche d'une identité qui lui permette de s'affirmer et de passer à l'âge adulte. Il y a ensuite son besoin de trouver une personne "admirable» et réconfortante, à l'instar d'une figure paternelle, et son rêve d'acquérir une notoriété, d'obtenir la reconnaissance des autres. Il faut encore noter l'intervention, hors-champ, de 
Lionel (Baier) qui interviewe Loïc sur ses ambitions, sa nouvelle passion pour la photographie... Il s'agit en quelque sorte d'une mise en abyme fictionalisée du parcours du réalisateur.

Son dernier film, Comme des voleurs, synthétise et affirme ces différentes relations entre fiction et documentaire de manière plus maîtrisée. Dans ce film, le cinéaste incarne un personnage portant le même prénom que lui; les éléments biographiques sont utilisés comme catalyseurs pour développer une histoire. Les origines polonaises de la famille Baier sont investies comme un prétexte exotique permettant au cinéaste de s'inventer une biographie fictive. Il y a également une bonne dose de jeu et d'espièglerie dans la démarche du réalisateur à interpréter un personnage homonyme. Où est la frontière entre la fiction et la biographie : qu'est-ce qui est vrai, qu'est-ce qui est faux?

"J'aime bien brouiller les pistes", explique Lionel Baier, "même si le spectateur se dit ‘ peut-être que c'est sa vie`, j’aime bien qu'il oublie cela et qu'il se retrouve juste dans le temps qui est celui du récit. L'important n'est pas de dire la vérité, mais quelque chose de vrai, non ?»1 Avec ce parti pris, il est difficile pour le spectateur de ne pas chercher à faire la part du vrai et du faux, à débrouiller les pistes, avant de se laisser entraîner par l'aventure. Ainsi Estocade, le roman d'autofiction écrit par le personnage de Lionel adolescent, donne une assise théorique au travail de Baier, sans que le film ne tente pour autant de faire accroire à l'existence de ce livre hors fiction (le dossier de presse, par exemple, ne le mentionne aucunement dans la bio-filmographie du réalisateur). Par son discours et à travers cette référence ponctuelle au roman autobiographique, le film revendique comme modèle l'autofiction, telle qu'elle a été pratiquée et théorisée par Serge Doubrovsky 2 : il s'agit en ce cas de transposer sa vie à travers une fiction irréalisante, tout en mobilisant le processus de la séance de la cure analytique ${ }^{3}$.

\section{L'aventure: une histoire de famille}

Le désir d'aventure traverse le film de part en part et se décline sous différentes formes. L'intérêt soudain du personnage de Lionel pour les origines polonaises de sa famille correspond à une envie de fuir la réalité et le quotidien. De même, sa sœur Lucie vit une période de crise et de remise en question et cherche à rompre avec ses habitudes. Passant d'abord par un rapport fantasmé aux origines polonaises, le voyage spirituel de Lionel se transforme dans la deuxième partie du film, grâce à l'impulsion de Lucie, en road movie bien ancré dans la réalité.

Dès le début de Comme des voleurs, après la séquence de la messe de Noël, Lionel, devant la porte de la maison familiale, écoute interloqué
1 Dossier de presse de Comme des voleurs (à l'Est).

2 Serge Doubrovsky proposait, dans son roman autobiographique Fils, la catégorie d'autofiction, qui a été depuis reprise ou critiquée régulièrement: "Autobiographie? Non, c'est un privilège réservé aux importants de ce monde, au soir de leur vie, et dans un beau style. Fiction, d'événements et de faits strictement réels; si l'on veut, autofiction, d'avoir confié le langage d'une aventure à l'aventure du langage, hors sagesse et hors syntaxe du roman, traditionnel ou nouveau. Rencontres, fils des mots, allitérations, assonances, dissonances, écriture d'avant ou après la littérature, concrète, comme on dit en musique. Ou encore, autofriction, patiemment onaniste, qui espère maintenant partager son plaisir." (Serge Doubrovsky, Fils, Galileee, Paris, 1977, quatrième de couverture.)

3 Dans un essai critique, Doubrovsky rapporte explicitement l'écriture d'autofiction à la séance psychanalytique: "L'autofiction, c'est la fiction que j'ai décidé, en tant qu'écrivain, de me donner à moi-même, en y incorporant, au sens plein du terme, l'expérience de l'analyse, non point seulement dans la thématique, mais dans la production du texte." (Serge Doubrovsky, "Autobiographie / vérité / psychanalyse", Autobiographies: de Corneille à Sartre, PUF, Paris, 1988, p. 70.) 
Lionel en compagnie de son ami Serge; puis avec Ewa, lors du pique-nique familial
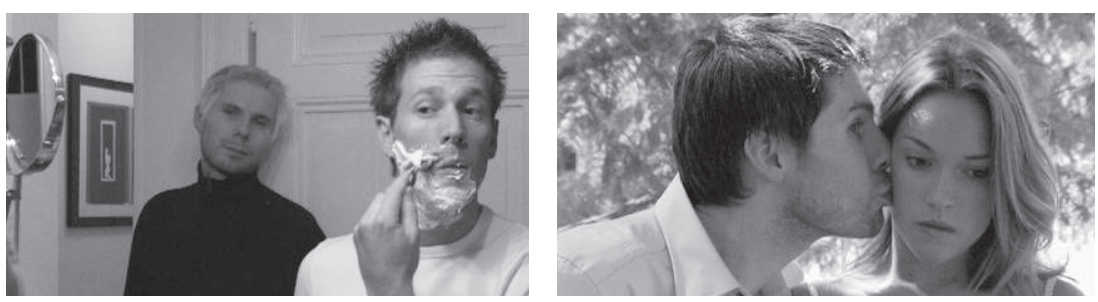

la proposition de sa sœur Lucie: elle l'invite à manquer le traditionnel repas et à partir avec elle à l'aventure, à "courir dans la nuit comme deux voleurs». Après un lourd silence, elle pousse finalement la porte d'entrée, prenant ainsi part à la soirée et renouant avec le cours réglé de sa vie. C'est finalement le père de Lionel qui répond à ses attentes lors d'une discussion en tête-à-tête, pendant laquelle il répond aux questions de son fils sur les origines polonaises de la famille. S'ensuit une recherche frénétique sur la Pologne: lecture de guides touristiques, cours de langue, rencontre avec une jeune fille au pair polonaise, Lionel saisit tous les moyens pour atteindre ce nouvel objectif: retrouver les traces de ce grand-père polonais.

L'attachement soudain de Lionel pour Ewa, la Polonaise, le conduit à une proposition inattendue de la part d'un jeune homme qui affirme son homosexualité. Sa demande en mariage pour permettre à cette dernière de rester en Suisse génère un certain nombre de situations comiques avant de devenir source de conflits. Son ami Serge, l'homme qui partage sa vie, d'abord amusé par le comportement excentrique de son ami, finit par se sentir trahi, déçu par ses actes excessifs. «Un souvenir contre une promesse", lit-il dans le journal intime de Lionel; cette formule lui ouvre brutalement les yeux sur la situation présente (lui-même étant relégué au statut d'une carte postale aux couleurs passées). Suite à l'emménagement d'Ewa chez Lionel et aux tensions que la situation a engendrées avec Serge, le couple s'installe chez Lucie. Bouleversée par le changement d'orientation sexuelle de son frère, leur relation devient également très tendue. Assistante sociale dans un centre de requérants d'asile, elle ne faisait que plaisanter en parlant de mariage à son frère et ne pensait en aucun cas que celui-ci la prendrait au mot... Seuls les parents apprennent la nouvelle avec plaisir, presque avec soulagement, au cours de l'inoubliable pique-nique familial qui se soldera par l'enlèvement de Lionel. Après avoir rejoint son frère dans la voiture, Lucie démarre en trombe, insensible à ses protestations et aux tentatives des autres protagonistes de l'arrêter. Excédée par les soudains revirements de comportements de son frère, Lucie décide de le confronter à la réalité polonaise. La deuxième partie du film s'ouvre ainsi sur un road movie par- 
semé d'embûches et de rencontres, traversées avec succès par ce nouveau couple, formé par Lionel et sa sœur. Au cours de ce voyage qui les mènera de Lausanne à Varsovie, le frère et la sœur traverseront de nombreuses épreuves, côtoieront la grande Histoire en arrivant à Auschwitz ${ }^{4}$, avant de parvenir à faire la lumière sur les origines polonaises de la famille.

Le voyage en Pologne fait également écho au livre du Suisse Blaise Cendrars, L'or (1925), que lit Lionel dès les premiers plans du film et qui donne lieu à une série de plans fantasmagoriques, aux couleurs saturées et irréelles. Un désir de transcender le quotidien et de s'y soustraire par l'imagination est donc présent dès les premières séquences. L'évocation fortuite avec le père des origines polonaises de la famille arrive donc à point pour permettre à Lionel d'exercer son imaginaire. Dans cette même perspective, on pense également au dialogue très littéraire entre Lucie et Lionel qui viennent d'arriver à Varsovie, après s'être fait voler leur voiture et leur argent. S'opposant au pessimisme et à au renoncement de Lucie, Lionel la convainc, au cours d'une longue tirade solennelle, de poursuivre leur aventure et de se laisser porter par l'inconnu. L'imaginaire et la réalité, la fiction et le documentaire, Lionel et Lucie, représentent deux forces opposées qui se côtoient tout le long du film et qui unissent leurs compétences pour réaliser un but commun.

\section{Vers un cinéma total}

Les films de Lionel Baier ont un autre point commun entre eux : la musique. En effet, tous font appel à des compositeurs classiques, Francis Poulenc pour Celui au pasteur, Camille de Saint-Saëns pour La parade, Sergei Rachmaninov pour Garçon stupide et Maurice Ravel pour Comme des voleurs. Le réalisateur accorde un soin tout particulier à accompagner ses images de musique symphonique. Ce choix n'est pas anodin, il souligne la charge émotionnelle du film. Baier, exploitant l'impact de la musique sur les images, plonge le spectateur dans une expérience audiovisuelle où la bande-son du film acquiert une importance toute particulière. Occupant au sein de l'univers filmographique la place d'un créateur démiurgique, le réalisateur manipule ses différents outils, agence les
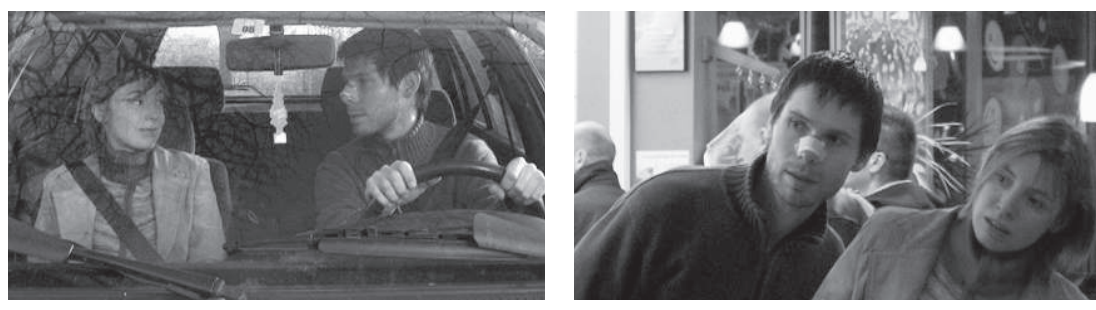
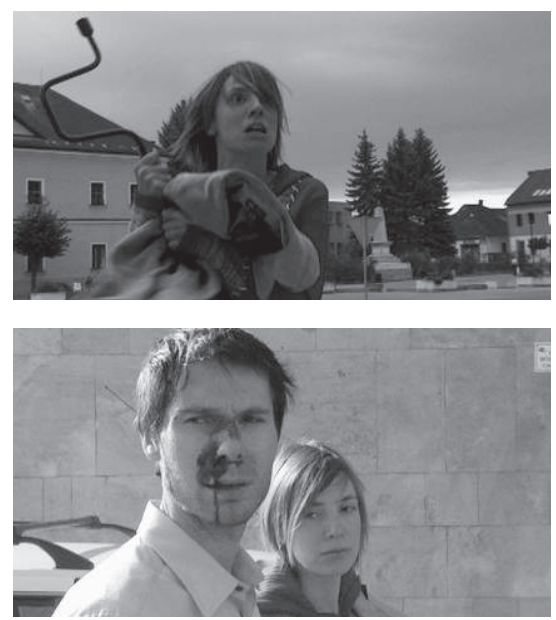

Lucie cherche à interrompre une dispute violente où un homme bat sa femme; Lionel après être intervenu

4 A noter également l'enchaînement des séquences qui encadrent cette scène: suite à l'intervention de Lucie lors d'une scène où un homme bat sa femme, Lionel se fait casser le nez en venant à la rescousse; l'arrivée en voiture sur un parking qui n'est autre que celui du musée d'Auschwitz, où défile une foule de touristes; et, finalement, la séquence dans le supermarché, d'où Lionel et sa sœur ressortent les bras chargés de victuailles... Humour décalé? Regard teinté de cynisme? Cette suite de clichés ne manque pas de soulever des interrogations.

Moments de complicité entre Lionel et sa sœur au cours de leur road movie 


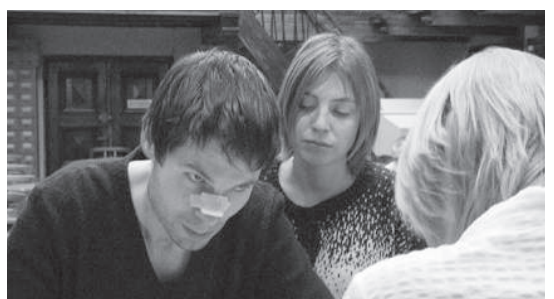

A la bibliothèque de Varsovie, quelques instants avant de retrouver l'aïeul tant recherché

5 Cité par Patrick d'Antan, $360^{\circ}$ magazine, novembre 2006.

6 Dossier de presse de Comme des voleurs (à l'Est).

$7 \mathrm{lbid}$. différentes composantes de son film suivant un schéma qui prend pour première cible le spectateur et sa sensibilité.

Baier considère le cinéma comme un bricolage et un art du faux, remettant en cause l'évidence, dénonçant comme artifice ce qui paraît aller de soi:

"Je trouve que la vie humaine est intéressante parce qu'elle transforme. On ment, on raconte des histoires, on trafique la vérité, on trafique la nature qui est autour de nous : mais je trouve que c'est plutôt une valeur humaine intéressante de trafiquer, de vouloir changer.» $\mathbf{5}$ S'octroyant le rôle principal dans son dernier film, Baier y est ainsi à la fois acteur et auteur, mêlant à nouveau les rôles. Des deux cotés de la caméra, il organise un monde dans lequel il peut transformer à sa guise la réalité qui l'entoure, faire des rencontres, aller à l'aventure et vivre des expériences dont il est le héros. Les différents comédiens qui l'entourent ne sont également pas tous des acteurs professionnels. Après de nombreux castings infructueux pour trouver l'interprète de Stan, l'ami polonais, Baier rencontre Michat Runnicki à la sortie d'un cours, à l'Alliance française de Varsovie, où il enseignait. De même, Luc André, artiste, peintre, photographe, enseignant et réalisateur de documentaires, vit sa première expérience en tant que comédien en jouant le rôle de Victor, le père de Lionel et Lucie. Ces acteurs non professionnels apportent au film leur présence singulière, non apprêtée, et viennent à nouveau troubler les limites entre fiction et documentaire. «J'aime qu'un personnage soit la rencontre de deux entités: une créature fictionnelle et un être bien réel», confirme Baier ${ }^{6}$. On peut encore noter la diversité des origines des membres de l'équipe, constituée de cinq nationalités différentes, et des lieux de tournage, réunissant cinq pays.

Les frontières, quelles qu'elles soient, sont là pour être franchies et permettre l'émergence de nouvelles expériences, qu'elles soient liées au jeu d'acteur, au mélange entre documentaire et fiction, à la composante sonore ou aux dimensions culturelle et géographique. A l'Est, comme l'indique le sous-titre du film, constitue un premier pas avant de poursuivre vers les autres point cardinaux. C'est que Comme des voleurs ( $\dot{a}$ l'Est) est le premier volet d'une tétralogie, nous apprend le réalisateur ${ }^{7}$, dont l'idée est de cartographier une Europe des sentiments. 